\title{
Genetic diversity and population structure of indigenous chicken breeds in South China
}

\author{
Xunhe HUANG (ه) ${ }^{1}$, Jinfeng ZHANG ${ }^{1}$, Danlin HE$^{2}$, Xiquan ZHANG ${ }^{2}$, Fusheng ZHONG ${ }^{1}$, Weina LI ${ }^{1}$, \\ Qingmei ZHENG ${ }^{1}$, Jiebo CHEN ${ }^{1}$, Bingwang DU $^{3}$ \\ 1 School of Life Sciences, Jiaying University, Meizhou 514015, China \\ 2 College of Animal Sciences, South China Agricultural University, Guangzhou 510642, China \\ 3 College of Agricultural, Guangdong Ocean University, Zhanjiang 524088, China
}

\begin{abstract}
A total of 587 individuals from 12 indigenous chicken breeds from South China and two commercial breeds were genotyped for 26 microsatellites to investigate the genetic diversity and population structure. All microsatellites were found to be polymorphic. The number of alleles per locus ranged from 5 to 36, with an average of $12.10 \pm 7.00$ (SE). All breeds, except White Recessive Rock, had high allelic polymorphism $(>0.5)$. Higher genetic diversity was revealed in the indigenous chicken breeds rather than in the commercial breeds. Potential introgression from the commercial breeds into the indigenous chickens was also detected. The population structure of these indigenous chicken breeds could be explained by their geographical distribution, which suggested the presence of independent history of breed formation. Data generated in this study will provide valuable information to the conservation for indigenous chicken breeds in future.
\end{abstract}

Keywords microsatellites, genetic diversity, population structure, indigenous chicken, South China, conservation

\section{Introduction}

Domestic chickens (Gallus gallus domesticus) are the most important poultry species providing humans with a stable source of protein ${ }^{[1]}$. The genetic diversity of indigenous chicken breeds is a valuable resource for breeding and understanding the extent of genetic diversity is important for resource conservation and molecular breeding ${ }^{[2-5]}$. There are abundant indigenous chickens especially the three-yellow (feather, skin and shank are yellow) breeds in South China. Recent studies of chickens in this region using microsatellites revealed high genetic diversity ${ }^{[6-8]}$.

Received March 18, 2016; accepted May 9, 2016

Correspondence: hxh826@jyu.edu.cn
However, due mostly to a paucity of sampled breeds ${ }^{[6-8]}$ and genotyped markers ${ }^{[6]}$, the patterns of genetic diversity distribution and population structure remain unclear. This research focused on reassessing the genetic diversity and population structure in indigenous chicken breeds from South China based on larger sample sizes as well as more microsatellites, with the aim of providing data to enable the design of conservation strategies for this valuable genetic resource.

\section{Materials and methods}

\subsection{Sampling and genotyping}

For this study, 537 individual birds were sampled from 12 indigenous chicken breeds from six provinces in South China. These breeds had been kept as broilers and/or layers usually in free-range management until about ten years ago. The blood samples from on average 45 randomly selected individuals per breed were collected from the conservation farms (Table 1). Additionally, two commercial breeds, Lingnan Yellow III (LN, $N=30)$ and White Recessive Rock (WR, $N=20$ ) were included in the study for comparison. LN are mixed with the lineage of Huiyang Bearded chicken and Guangxi Yellow chicken, and are largely raised in Guangdong, while WR, which were introduced from Israel, are usually used to assistant breeding in China.

Total genomic DNA was extracted from blood using the standard phenol-chloroform method. All birds were genotyped for the 26 autosomal microsatellite loci recommended by ISAG/FAO advisory group for chicken biodiversity (Appendix A, Table S1). Genotypes were determined on an ABI PRISM 3730 automated capillary sequencer (Applied Biosystems, Foster City, CA, USA) using LIZ500 as internal size standard in each lane, and scored using GeneMarker 2.2.0 (SoftGenetics, State College, USA) coupled with visual examinations. 


\subsection{Statistical analysis}

The mean number of alleles per locus (MNA), observed heterozygosity $\left(H_{\mathrm{O}}\right)$, expected heterozygosity $\left(H_{\mathrm{E}}\right)$ and inbreeding coefficients $\left(F_{\text {IS }}\right)$ were calculated with the FSTAT2.9.3.2 $2^{[9]}$. Given that the amount of observed allelic diversity can depend heavily on sample size $\mathrm{e}^{[10]}$, the program $\mathrm{ADZE}^{[11]}$ was used to compute rarified allelic richness $[\operatorname{Ar}(g)]$ and private allelic richness $[A p(g)]$ controlled for sample size, with $g$ representing the minimum number of individuals in one of the samples. Population structure was determined by the Bayesian model-based clustering as implemented in the STRUCTURE2.3.3 software ${ }^{[12,13]}$ and referred to the strategy from Evanno et al. ${ }^{[14]}$. Individuals were grouped into a predefined number of $K$ clusters $(1 \leqslant K \leqslant 15)$, with 10 independent runs for each $K$ value. The analysis was used a burn-in of 20000 followed by 50000 Markov Chain Monte Carlo (MCMC) iterations, under admixture and allele frequency correlations model. Results of the 10 STRUCTURE runs were obtained using Structure Harvester ${ }^{[15]}$ and then merged with CLUMPP1.1.2 ${ }^{[16]}$, and finally visualized with DISTRUCT1.1 ${ }^{[17]}$. The factorial correspondence analysis implemented in GENETIX $4.05^{[18]}$ was performed to reveal the relationship between chicken breeds.

\section{Results}

A total of 314 alleles were detected at 26 microsatellite loci across 587 individuals from 14 chicken breeds. The alleles ranged from 5 (MCW0165 and MCW0222) to 36 (LEI0234) per locus with an average of $12.10 \pm 7.00$ (Appendix A, Table S2). All breeds except WR showed high allelic polymorphism $(>0.5)$, ranging from $0.64 \pm$ 0.16 and $0.61 \pm 0.16$ to $0.70 \pm 0.13$ and $0.68 \pm 0.18$ for $H_{\mathrm{E}}$ and $H_{\mathrm{O}}$, respectively. $\mathrm{HL}$ and $\mathrm{WR}$ breeds had the highest $(8.27 \pm 4.53)$ and lowest $(3.69 \pm 1.50)$ value of the mean number of alleles, respectively. Given that the allelic richness was dependent on the sample size, when the mean number of private alleles and allelic richness rarified to a sample size of 20, allelic diversity between breeds did not differ significantly, but indigenous breeds possessed higher genetic diversity and lower $F_{\text {IS }}$ than commercial breeds did (Table 1).

The Bayesian ancestry inference using STRUCTURE indicated the presence of substructure in these indigenous

Table 1 Genetic diversity of 12 indigenous chicken breeds from South China and two commercial breeds using 26 microsatellite markers

\begin{tabular}{|c|c|c|c|c|c|c|c|c|c|}
\hline \multirow{2}{*}{ Breed (code) } & \multirow{2}{*}{$N$} & \multicolumn{4}{|c|}{ Allelic diversity } & \multicolumn{2}{|c|}{ Genetic diversity } & \multicolumn{2}{|c|}{ Proportion of clusters } \\
\hline & & TNA & $\mathrm{MNA} \pm \mathrm{SE}$ & $\operatorname{Ar}(g) \pm \mathrm{SE}$ & $A p(g) \pm \mathrm{SE}$ & $H_{\mathrm{E}} \pm \mathrm{SE}$ & $H_{\mathrm{O}} \pm \mathrm{SE}$ & $\mathrm{I} / \mathrm{II} / \mathrm{III}$ & $F_{\text {IS }}$ \\
\hline Wenchang (WC)* & 34 & 183 & $7.04 \pm 3.45$ & $5.21 \pm 0.37$ & $0.13 \pm 0.03$ & $0.70 \pm 0.13$ & $0.65 \pm 0.15$ & $0.90 / 0.04 / 0.06$ & 0.08 \\
\hline Guangxi Yellow (GX)* & 32 & 172 & $6.62 \pm 3.29$ & $4.85 \pm 0.40$ & $0.13 \pm 0.04$ & $0.65 \pm 0.16$ & $0.65 \pm 0.21$ & $0.94 / 0.02 / 0.04$ & 0.01 \\
\hline Huaixiang $(\mathrm{HX})^{*}$ & 78 & 209 & $8.04 \pm 4.47$ & $5.17 \pm 0.41$ & $0.14 \pm 0.04$ & $0.67 \pm 0.14$ & $0.66 \pm 0.16$ & $0.92 / 0.03 / 0.05$ & 0.03 \\
\hline Xinghua $(\mathrm{XH})^{*}$ & 36 & 156 & $6.00 \pm 2.79$ & $4.65 \pm 0.35$ & $0.06 \pm 0.03$ & $0.64 \pm 0.16$ & $0.67 \pm 0.17$ & $0.85 / 0.03 / 0.12$ & -0.03 \\
\hline Zhongshan Shalan (SL)* & 36 & 171 & $6.58 \pm 3.26$ & $4.83 \pm 0.40$ & $0.11 \pm 0.05$ & $0.65 \pm 0.14$ & $0.68 \pm 0.17$ & $0.96 / 0.02 / 0.02$ & -0.04 \\
\hline Qingyuan Partridge (QY)* & 47 & 190 & $7.31 \pm 3.25$ & $4.99 \pm 0.38$ & $0.11 \pm 0.03$ & $0.65 \pm 0.15$ & $0.67 \pm 0.18$ & $0.94 / 0.02 / 0.04$ & -0.02 \\
\hline Yangshan (YS)* & 36 & 166 & $6.38 \pm 3.07$ & $4.77 \pm 0.35$ & $0.06 \pm 0.03$ & $0.65 \pm 0.16$ & $0.66 \pm 0.20$ & $0.87 / 0.04 / 0.09$ & 0.00 \\
\hline Huanglang (HL)* & 50 & 215 & $8.27 \pm 4.53$ & $5.35 \pm 0.41$ & $0.24 \pm 0.05$ & $0.68 \pm 0.14$ & $0.68 \pm 0.18$ & $0.89 / 0.02 / 0.09$ & 0.02 \\
\hline Huiyang Bearded (HY)* & 35 & 171 & $6.58 \pm 2.73$ & $4.97 \pm 0.29$ & $0.15 \pm 0.04$ & $0.69 \pm 0.09$ & $0.64 \pm 0.13$ & $0.05 / 0.90 / 0.05$ & 0.08 \\
\hline Wuhua Yellow (WH) & 58 & 191 & $7.35 \pm 3.27$ & $4.95 \pm 0.30$ & $0.13 \pm 0.03$ & $0.69 \pm 0.11$ & $0.65 \pm 0.15$ & $0.04 / 0.92 / 0.04$ & 0.06 \\
\hline Ningdu Yellow (ND)* & 50 & 181 & $6.96 \pm 3.74$ & $4.94 \pm 0.40$ & $0.17 \pm 0.06$ & $0.67 \pm 0.14$ & $0.61 \pm 0.16$ & $0.25 / 0.02 / 0.73$ & 0.10 \\
\hline Hetian $(\mathrm{HT})^{*}$ & 45 & 181 & $6.96 \pm 3.90$ & $5.05 \pm 0.42$ & $0.16 \pm 0.05$ & $0.68 \pm 0.14$ & $0.62 \pm 0.18$ & $0.04 / 0.04 / 0.92$ & 0.10 \\
\hline Lingnan Yellow III (LN) & 30 & 177 & $6.81 \pm 3.74$ & $5.32 \pm 0.41$ & $0.14 \pm 0.04$ & $0.70 \pm 0.13$ & $0.63 \pm 0.20$ & $0.59 / 0.12 / 0.29$ & 0.11 \\
\hline White Recessive Rock (WR) & 20 & 96 & $3.69 \pm 1.50$ & $3.31 \pm 0.24$ & $0.04 \pm 0.02$ & $0.48 \pm 0.18$ & $0.48 \pm 0.20$ & $0.01 / 0.88 / 0.11$ & 0.05 \\
\hline Cluster I & 349 & 281 & $10.81 \pm 6.34$ & $8.02 \pm 0.84$ & $0.93 \pm 0.19$ & $0.68 \pm 0.14$ & $0.66 \pm 0.15$ & $0.90 / 0.04 / 0.06$ & 0.03 \\
\hline Cluster II & 93 & 220 & $8.46 \pm 4.12$ & $7.53 \pm 0.66$ & $0.80 \pm 0.14$ & $0.71 \pm 0.10$ & $0.65 \pm 0.13$ & $0.06 / 0.61 / 0.33$ & 0.08 \\
\hline Cluster III & 95 & 214 & $8.23 \pm 4.73$ & $7.43 \pm 0.80$ & $0.78 \pm 0.18$ & $0.69 \pm 0.14$ & $0.61 \pm 0.15$ & $0.02 / 0.04 / 0.94$ & 0.11 \\
\hline Commercial breeds & 50 & 188 & $7.23 \pm 3.81$ & $6.38 \pm 0.57$ & $0.97 \pm 0.27$ & $0.69 \pm 0.11$ & $0.57 \pm 0.13$ & $0.32 / 0.50 / 0.18$ & 0.17 \\
\hline Indigenous breeds & 537 & 310 & $11.92 \pm 6.77$ & $7.20 \pm 0.67$ & $1.80 \pm 0.36$ & $0.70 \pm 0.13$ & $0.65 \pm 0.13$ & $0.60 / 0.19 / 0.21$ & 0.07 \\
\hline Overall & 587 & 314 & $12.10 \pm 7.00$ & - & - & $0.71 \pm 0.12$ & $0.65 \pm 0.13$ & $0.63 / 0.32 / 0.05$ & 0.09 \\
\hline
\end{tabular}

Note: $N$, sample size; TNA, total number of alleles; MNA, mean number of alleles; $\operatorname{Ar}(g)$ and $\operatorname{Ap}(g)$, allelic and private allelic richness, respectively, with rarefaction for a corresponding sample size of 20 for all samples, 93 for Cluster I, II and III, 50 for commercial and indigenous breeds; $H_{\mathrm{E}}$, expected heterozygosity; $H_{\mathrm{O}}$, observed heterozygosity; SE, standard error; $F_{\text {IS }}$, coefficient of inbreeding; *, breeds are recorded in Animal Genetic Resources in China: Poultry (China National Commission of Animal Genetic Resources 2011). 
chicken breeds. The value of $\Delta K$ at $K=2$ was much larger than that at $K=3$ or other values (Appendix B, Fig. S1). The height of the modal values of $\Delta K$ indicates the strength of the population subdivision signal ${ }^{[14]}$. At $K=2,14$ chicken breeds were divided into two clusters (WC, GX, HX, SL, XH, QY, YS, HL and LN vs. HY, WH, ND, HT and WR) (Fig. 1a). However, at $K=3$, the second cluster further separated to two subclusters (HY, WH and YX vs. ND and HT, respectively) (Fig. 1a). Furthermore, at $K>3$, there was no change in the genetic structure (Fig. 1a). Consulting the suggestion from Pritchard et al. ${ }^{[12]}$ and Mwacharo et al. ${ }^{[19]}$, as well as geographic information for the chicken breeds studied, $K=3$ was chosen as the most optimal number of genetic clusters to reveal the biologically meaningful genetic structure of indigenous chicken breeds. For brevity, these three clusters of indigenous chicken breeds are referred to as cluster I (green), II (purple) and III (red) (Table 1, Fig. 1). Overall, the clusters of 12 current chicken populations in Bayes structure related to their geographical localization (Fig. 1b). Also, the clustering pattern at $K=3$ corresponded to the result in factorial correspondence analysis (Appendix B, Fig. S2).

\section{Discussion}

The genetic diversity and structure of 12 indigenous chicken breeds from South China and two commercial breeds were evaluated used 26 microsatellite loci. All loci were found to be polymorphic, which confirmed that these markers were appropriate for evaluating the genetic diversity. Our results indicated the indigenous breeds from South China harbor higher levels of genetic diversity, as compared with the commercial breeds and were generally in agreement with previous studies ${ }^{[6-8]}$, although with a few difference in mean number of alleles per locus or heterozygosity. The lower genetic diversity in the commercial breeds could be attributed to the intense artificial selection over a short period of time and a limited effective flock size ${ }^{[20]}$.
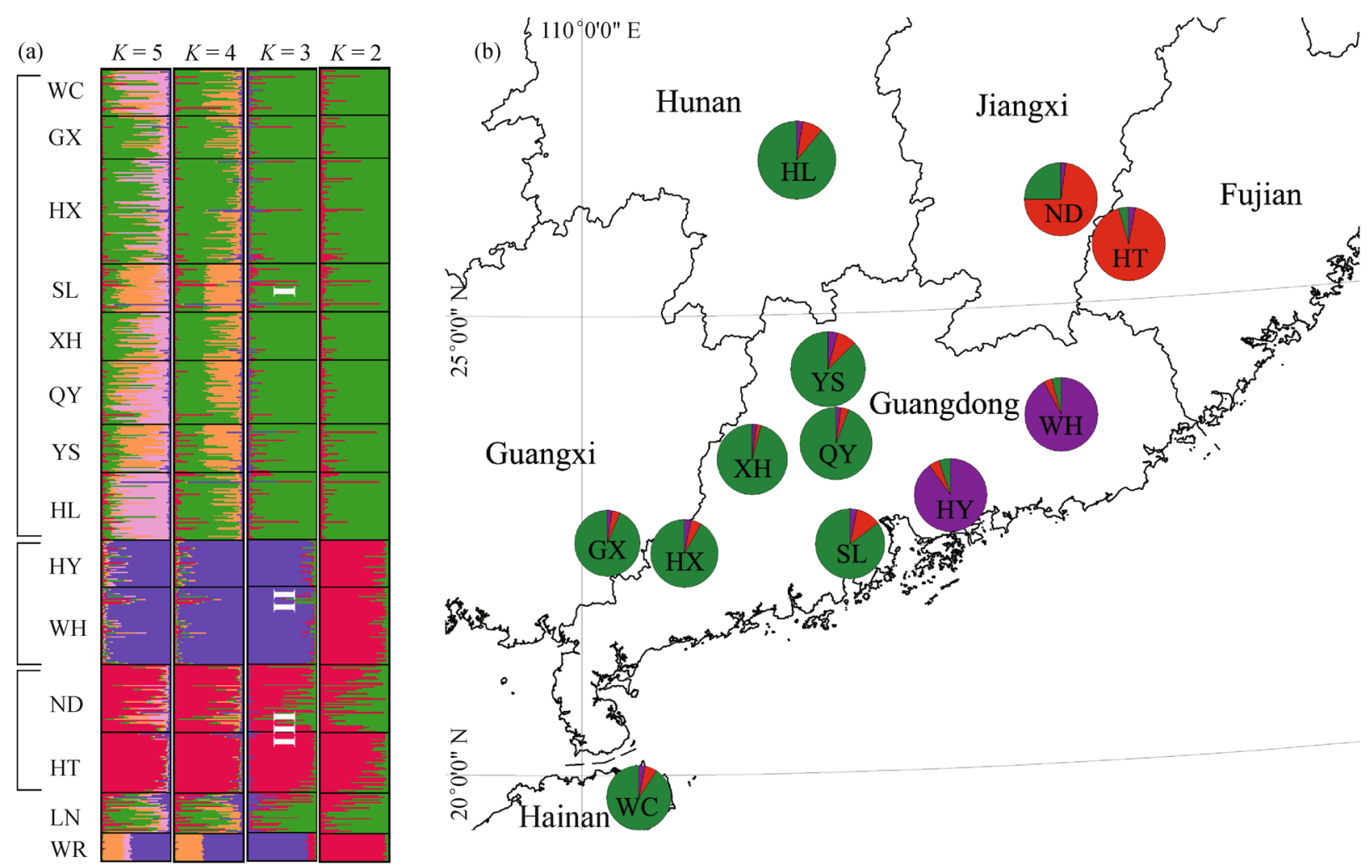

Fig. 1 (a) Substructure of 12 indigenous chicken breeds from South China and commercial breeds as indicated by STRUCTURE analysis. Each individual represented by a single vertical line was assigned to one of three distinct clusters based on clustering result at $K=$ 3. Black bars are separators fo r breeds; Color codes: green, Cluster I; purple, Cluster II; red, Cluster III(b) geographical distribution of 12 indigenous chicken breeds from South China. The shaded area in each pie chart is proportional to the number of individuals in each population observed for each genetic cluster. Color codes: green, Cluster I; purple, Cluster II; red, Cluster III. Breed codes: WC, Wenchang; GX, Guangxi Yellow; HX, Huaixiang; SL, Zhongshan Shalan; XH, Xinghua; QY, Qingyuan Partridge; YS, Yangshan; HL, Huanglang; HY, Huiyang Bearded; WH, Wuhua Yellow; ND, Ningdu Yellow; HT, Hetian; LN, Lingnan Yellow III; WR, White Recessive Rock. 
The genetic structures in these chicken breeds corresponded to their geographical localization. Indeed, the economically developed areas of South China in particular have had prolonged and sustained socioeconomic interactions with each other. Such interactions have facilitated the gene flow among indigenous chicken breeds, especially those in adjacent geographical regions, and between indigenous ${ }^{[19,21]}$. Different allelic proportions in these three clusters suggested the presence of an independent history of breed formation. This possibility needs further investigation with a larger number of breeds from more diverse locations and more molecular markers, such as mitochondrial DNA and high-density SNP arrays.

It should be noted that the genetic cluster of WR was similar to HY and WH (Fig. 1a, $K=3$ ). This is expected to result from introgression because WR was recently imported for breeding with indigenous chickens in China. For example, some broiler breeds used crosses with Cochin and Langshan that were very closely related to HY, XH and QY. More importantly, these are meat breeds and probably had a similar genetic origin ${ }^{[6]}$. Although introgression between chicken breeds, and between domestic chicken and wild junglefowl has been previously reported $^{[21-24]}$, further research needs to be initiated to confirm this hypothesis.

\section{Conclusions}

The results presented here are the first systematic report on the genetic diversity and structure of indigenous chicken breeds from South China. Overall, indigenous chicken breeds had high levels of significant genetic diversity and structure. Also, potential introgression from the commercial breeds into the indigenous chickens was detected. These results can be used as baseline genetic information for genetic conservation programs, for instance, avoidance of inbreeding and introgression.

Acknowledgements We sincerely thank Zhiguan ZOU and Shaofeng LIU for providing HY and GX samples, respectively, and Kunfeng Tan of Jiaying University for experimental assistance. We also thank Dr. Minsheng PENG of Kunming Institute of Zoology, Chinese Academy of Sciences for constructive comments on the manuscript. This research was supported by Guangdong Natural Science Foundation (2014A030307018), Innovation and strong school project of Jiaying University (CQX019), Outstanding Young Teacher Training Program of Colleges and Universities in Guangdong Province (Yq2013152), Science and Technology Planning Project of Guangdong Province (2015A020208020, 2016A030303068).

Supplementary materials The online version of this article at $\mathrm{http}: / / \mathrm{dx}$. doi.org/10.15302/J-FASE-2016102 contains supplementary materials (Appendixes A and B).

Compliance with ethics guidelines Xunhe Huang, Jinfeng Zhang, Danlin He, Xiquan Zhang, Fusheng Zhong, Weina Li, Qingmei Zheng, Jiebo Chen, and Bingwang Du declare that they have no conflict of interest or financial conflicts to disclose.

All applicable institutional and national guidelines for the care and use of animals were followed.

\section{References}

1. FAO. The State of the World's Animal Genetic Resources for Food and Agriculture. Rome: FAO, 2007

2. Blackburn $\mathrm{H}$ D. The national animal germplasm program: challenges and opportunities for poultry genetic resources. Poultry Science, 2006, 85(2): 210-215

3. Toro M A, Fernández J, Caballero A. Molecular characterization of breeds and its use in conservation. Livestock Science, 2009, 120(3): 174-195

4. Lyimo C M, Weigend A, Msoffe P L, Eding H, Simianer H, Weigend S. Global diversity and genetic contributions of chicken populations from African, Asian and European regions. Animal Genetics, 2014, 45(6): 836-848

5. Ceccobelli S, Di Lorenzo P, Lancioni H, Monteagudo Ibáñez L V, Tejedor M T, Castellini C, Landi V, Martínez Martínez A, Delgado Bermejo J V, Vega Pla J L, Leon Jurado J M, García N, Attard G, Grimal A, Stojanovic S, Kume K, Panella F, Weigend S, Lasagna E. Genetic diversity and phylogeographic structure of sixteen Mediterranean chicken breeds assessed with microsatellites and mitochondrial DNA. Livestock Science, 2015, 175: 27-36

6. Zhang X Q, Leung F C, Chan D K O, Yang G, Wu C. Genetic diversity of Chinese native chicken breeds based on protein polymorphism, randomly amplified polymorphic DNA, and microsatellite polymorphism. Poultry Science, 2002, 81(10): 1463-1472

7. Qu L J, Li X Y, Xu G F, Chen K W, Yang H J, Zhang L C, Wu G Q, Hou Z C, Xu G Y, Yang N. Evaluation of genetic diversity in Chinese indigenous chicken breeds using microsatellite markers. Science in China. Series C: Life Sciences, 2006, 49(4): 332-341

8. Yu Y B, Wang J Y, Mekki D M, Tang Q P, Li H F, Gu R, Ge Q L, Zhu W Q, Chen K W. Evaluation of genetic diversity and genetic distance between twelve Chinese indigenous chicken breeds based on microsatellite markers. International Journal of Poultry Science, 2006, 5(6): 550-556

9. Goudet J. FSTAT, a program to estimate and test gene diversities and fixation indices (version 2.9.3.2). http://www2.unil.ch/popgen/softwares/fstat.htm, 2016-01-05

10. Leberg P L. Estimating allelic richness: effects of sample size and bottlenecks. Molecular Ecology, 2002, 11(11): 2445-2449

11. Szpiech Z A, Jakobsson M, Rosenberg N A. ADZE: a rarefaction approach for counting alleles private to combinations of populations. Bioinformatics, 2008, 24(21): 2498-2504

12. Pritchard J K, Stephens M, Donnelly P. Inference of population structure using multilocus genotype data. Genetics, 2000, 155(2): 945-959

13. Hubisz M J, Falush D, Stephens M, Pritchard J K. Inferring weak population structure with the assistance of sample group information. Molecular Ecology Resources, 2009, 9(5): 1322-1332

14. Evanno G, Regnaut S, Goudet J. Detecting the number of clusters of individuals using the software STRUCTURE: a simulation study. Molecular Ecology, 2005, 14(8): 2611-2620

15. Earl D A, vonHoldt B M. STRUCTURE HARVESTER: a website and program for visualizing STRUCTURE output and implementing the Evanno method. Conservation Genetics Resources, 2012, 4 (2): 359-361

16. Jakobsson M, Rosenberg N A. CLUMPP: a cluster matching and 
permutation program for dealing with label switching and multimodality in analysis of population structure. Bioinformatics, 2007, 23(14): 1801-1806

17. Rosenberg N A. DISTRUCT: a program for the graphical display of population structure. Molecular Ecology Notes, 2004, 4(1): 137-138

18. Belkhir K, Borsa P, Chikhi L, Raufaste N, Bonhomme F. GENETIX 4.05, Logiciel Sous Windows TM pour la Génétique des Populations. http://kimura.univ-montp2.fr/genetix/, 2015-12-5

19. Mwacharo J M, Nomura K, Hanada H, Han J L, Amano T, Hanotte O. Reconstructing the origin and dispersal patterns of village chickens across East Africa: insights from autosomal markers. Molecular Ecology, 2013, 22(10): 2683-2697

20. Groeneveld L F, Lenstra J A, Eding H, Toro M A, Scherf B, Pilling D, Negrini R, Finlay E K, Jianlin H, Groeneveld E, Weigend S. Genetic diversity in farm animals - a review. Animal Genetics, 2010, 41(S1): 6-31

21. Mahammi F Z, Gaouar S B S, Laloë D, Faugeras R, Tabet-Aoul N, Rognon X, Tixier-Boichard M, Saidi-Mehtar N. A molecular analysis of the patterns of genetic diversity in local chickens from western Algeria in comparison with commercial lines and wild jungle fowls. Journal of Animal Breeding and Genetics, 2016, 133 (1): 59-70

22. Mwacharo J M, Bjørnstad M, Mobegi V, Nomura K, Hanada H, Amano T, Han J L, Hanotte O. Mitochondrial DNA reveals multiple introductions of domestic chicken in East Africa. Molecular Phylogenetics and Evolution, 2011, 58(2): 374-382

23. Leroy G, Kayang B B, Youssao I A K, Yapi-Gnaoré C V, OseiAmponsah R, Loukou N G E, Fotsa J C, Benabdeljelil K, Bed'hom $\mathrm{B}$, Tixier-Boichard M, Rognon X. Gene diversity, agroecological structure and introgression patterns among village chicken populations across North, West and Central Africa. BMC Genetics, 2012, 13(1): 34

24. Gering E, Johnsson M, Willis P, Getty T, Wright D. Mixed ancestry and admixture in Kauai's feral chickens: invasion of domestic genes into ancient Red Junglefowl reservoirs. Molecular Ecology, 2015, 24(9): 2112-2124 